\title{
ON A CLASS OF DISTRIBUTION FUNCTIONS WHERE THE QUOTIENT FOLLOWS THE CAUCHY LAW( $\left.{ }^{1}\right)$
}

\author{
BY \\ R. G. LAHA
}

1. Introduction. Let $x$ and $y$ be two independent normal variates distributed with zero means and a common variance; then it is well-known that the quotient $x / y$ follows the Cauchy law distributed symmetrically about the origin. Now the question that naturally arises is whether it is possible to obtain a characterization of the normal distribution by this property of the quotient. The converse problem can be more precisely formulated as follows:

Let $x$ and $y$ be two independently and identically distributed random variables having a common distribution function $F(x)$. Let the quotient $\omega=x / y$ follow the Cauchy law distributed symmetrically about the origin $\omega=0$. Then the question that arises is whether $F(x)$ is normal.

But this converse problem is not true; a very simple example of a nonnormal distribution $F(x)$ where the quotient $x / y$ follows the Cauchy law has been constructed by the author in [1]. Thus our problem now essentially reduces to that of complete enumeration of the class of distribution laws $F(x)$ where the quotient $x / y$ follows the Cauchy law. The author [2] has also derived some interesting general properties possessed by the class of distribution functions $F(x)$. Recently Steck [7] has also given some examples of nonnormal distributions $F(x)$ where the quotient has a Cauchy law $\left({ }^{2}\right)$. In the present paper we shall discuss some methods of construction of a class of distribution functions $F(x)$ having finite absolute moments up to a certain order $k(k \geqq 1)$ where the quotient $x / y$ has the Cauchy law. In $\$ 2$ we derive some useful analytical lemmas. In $\$ 3$ we make use of these lemmas and construct a class of distribution functions $F(x)$. In the final section we apply some results in the theory of rational characteristic functions and non-negative trigonometric polynomials and construct another class of distribution functions $F(x)$.

2. Some useful lemmas. We now prove some lemmas which are to be deduced essentially from the theory of analytic functions. These lemmas are

Received by the editors September 16, 1958.

(1) This research was sponsored by the National Science Foundation, Grant NSF G 4220, and by the Office of Naval Research under Contract Number Nonr-266(33), Project Number NR 042-034. Reproduction in whole or in part is permitted for any purpose of the United States Government.

(2) Note added in proof. While this paper was in press, the author has learnt in a communication that some examples of nonnormal distributions were also constructed by J. G. Mauldon in Quart. J. Math. Oxford ser. (2) vol. 7 (1956) pp. 155-160. 
useful in the proofs of the subsequent results and are also of independent interest.

LEMMA 2.1. Let $f(x)$ be a continuous non-negative function of the real variable $x$. Let the integral

$$
\int_{0}^{\infty} x^{v} f(x) d x
$$

exist for all $v$ in the interval $0 \leqq v \leqq V,(V>0)$. Then the integral

$$
I(z)=\int_{0}^{\infty} x^{-i z} f(x) d x \quad(z \text { a complex number })
$$

is an analytic function of the complex variable $z$ which is regular at least in the strip $0 \leqq \operatorname{Im}(z) \leqq V$ of the upper half plane.

Proof. We write the integral $I(z)$ as the sum of $\Phi_{1}(z)$ and $\Phi_{2}(z)$ such that

$$
I(z)=\Phi_{1}(z)+\Phi_{2}(z)
$$

where

$$
\Phi_{1}(z)=\int_{0}^{1} x^{-i z} f(x) d x ; \quad \Phi_{2}(z)=\int_{1}^{\infty} x^{-i z} f(x) d x .
$$

We first discuss the analyticity of the function $\Phi_{2}(z)$. Let us suppose that $z$ ranges over an arbitrary bounded region $D$ which is contained in the strip $0 \leqq \operatorname{Im}(z) \leqq V$ of the upper half plane. Then there exist two real constants $\gamma$ and $\delta$ such that

$$
\gamma \leqq \operatorname{Im}(z) \leqq \delta \quad(0 \leqq \gamma<\delta \leqq V) .
$$

Now putting $z=u+i v$ ( $u$ and $v$ both real), we see easily that whenever $z$ lies in this arbitrary bounded region $D$, we have

$$
\left|x^{-i z}\right|=x^{v} \leqq x^{\delta}
$$

provided that $x \geqq 1$. Thus for all values of $z$ in $D$, we have

$$
\left|\Phi_{2}(z)\right| \leqq \int_{1}^{\infty} x^{\delta} f(x) d x .
$$

Then using the test of uniform convergence [8, p. 22] it follows at once from (2.2) that $\Phi_{2}(z)$ is uniformly convergent in the same region $D$. Finally applying the definition of analytic functions by means of integrals [8, pp. 99-100], we deduce easily that $\Phi_{2}(z)$ is an analytic function of the complex variable $z$ which is regular at least in the strip $0 \leqq \operatorname{Im}(z) \leqq V$ of the upper half plane.

In the same way, we derive the analyticity of the integral $\Phi_{1}(z)$. When $z$ lies in the same arbitrary bounded region $D$, we can show easily that 


$$
\left|\Phi_{1}(z)\right| \leqq \int_{0}^{1} x^{\gamma} f(x) d x .
$$

From (2.3), repeating the same argument as in the case of $\Phi_{2}(z)$, we prove that $\Phi_{1}(z)$ is also an analytic function which is regular at least in the same strip $0 \leqq \operatorname{Im}(z) \leqq V$ of the upper half plane. This completes the proof of the lemma.

As a special case of this lemma, we can verify easily that if the integral $\int_{0}^{\infty} x^{v} f(x) d x$ exists for all $v \geqq 0$, then $I(z)$ is regular throughout the upper half plane $\operatorname{Im}(z) \geqq 0$.

We next prove some analogous lemmas in the theory of probability which are themselves of independent interest.

LemMa 2.2. Let the distribution function $F(x)$ of a random variable $x$ be symmetric about the origin $x=0$, absolutely continuous and have a continuous probability density function $f(x)=F^{\prime}(x)$ and also possess finite absolute moments $u p$ to a certain order $k(k \geqq 1)$. Let $\phi(t)=E\left(e^{i t \log |x|}\right)$ denote the characteristic function of the distribution of $\log |x|$. Then the function

$$
\phi(-z)=E\left(e^{-i z \log |x|}\right)
$$

considered as a function of the complex variable $z$, is an analytic function which is regular at least in the strip $0 \leqq \operatorname{Im}(z) \leqq V$ of the upper half plane (where $k \leqq V<k+1)$.

Proof.- Using the fact that the distribution function $F(x)$ is symmetric and absolutely continuous with a continuous probability density function $f(x)$, we obtain

$$
\phi(-z)=\int_{-\infty}^{\infty} e^{-i z \log |x|} f(x) d x=2 \int_{0}^{\infty} x^{-i z} f(x) d x .
$$

As the distribution function $F(x)$ has finite absolute moments up to order $k(k \geqq 1)$, we see easily that the integral

$$
\int_{0}^{\infty} x^{v} f(x) d x
$$

exists for all $v$ in the interval $0 \leqq v \leqq V$ where $k \leqq V<k+1$. Then the proof follows at once from Lemma 2.1 .

As a special case of Lemma 2.2 , it can be easily verified that if $F(x)$ has finite moments of all orders, then $\phi(-z)=E\left(e^{-i z \log |x|}\right)$ is an analytic function of the complex variable $z$ regular in the upper half plane $\operatorname{Im}(z) \geqq 0$.

Leмma 2.3. Let $x$ and $y$ be two independently and identically distributed random variables having a common distribution function $F(x)$. Let the quotient $\omega=x / y$ follow the Cauchy law distributed symmetrically about the origin $\omega=0$. 
Let further $F(x)$ have finite absolute moments up to a certain order $k(k \geqq 1)$. If $\phi(t)=E\left(e^{i t \log |x|}\right)$ denotes the characteristic function of the distribution of $\log |x|$, then the function

$$
\phi(-z)=E\left(e^{-i z \log |x|}\right),
$$

considered as a function of the complex variable $z$, is an analytic characteristic function which is regular in the strip $-1<\operatorname{Im}(z)<k+1$ containing the real axis.

Proof. From [2] where the author has studied some general properties of such distribution laws $F(x)$, it follows under the conditions of Lemma 2.3, that $F(x)$ is also symmetric about the origin $x=0$, absolutely continuous and has a continuous probability density function $f(x)=F^{\prime}(x)$. It is given that $\omega=x / y$ follows the Cauchy law distributed symmetrically about the origin $\omega=0$. Thus we can easily verify after elementary integration that the characteristic function of the distribution of $\log |\omega|$ is given by

$$
E\left(e^{i t \log |\omega|}\right)=\frac{1}{\cosh \left(\frac{\pi \downarrow}{2}\right)} .
$$

Finally noting the relation

$$
\log |\omega|=\log |x|-\log |y|,
$$

we get easily the relation

$$
\phi(t) \phi(-t)=\frac{1}{\cosh \left(\frac{\pi t}{2}\right)},
$$

holding for all real $t$, where $\phi(t)$ denotes the characteristic function of the distribution of $\log |x|$. Next we note that the characteristic function $1 / \cosh (\pi t / 2)$ can be continued in the complex $z$-plane such that $1 / \cosh (\pi z / 2)$ is an analytic characteristic function which is regular in the strip $|\operatorname{Im}(z)|<1$. Then using the theorem of Lévy [3] and Raikov [6, pp. 98-99] on the decomposition of analytic characteristic functions, we see easily from the relation (2.5) that $\phi(t)$ can also be continued in the complex z-plane and further $\phi(-z)$ is also regular at least in the same strip $|\operatorname{Im}(z)|<1$.

Again since $F(x)$ has finite absolute moments up to order $k$, it follows at once from Lemma 2.2 that $\phi(-z)=E\left(e^{-i z \log |x|}\right)$ is an analytic function which is regular at least in the strip $0 \leqq \operatorname{Im}(z) \leqq V$ (where $k \leqq V<k+1$ ). Thus combining these two results, we conclude that $\phi(-z)$ is an analytic characteristic function which is regular in the strip $-1<\operatorname{Im}(z)<k+1$ containing the real axis. Hence the proof. 
3. Construction of a class of distribution laws. In this section we discuss the method of construction of a class of infinitely many distribution functions $F(x)$ where the quotient follows the Cauchy law. For this purpose we examine more closely the equation

$$
\phi(z) \phi(-z)=\frac{1}{\cosh (\pi z / 2)}
$$

holding for all complex $z$ in the strip $|\operatorname{Im}(z)|<1$. From (3.1) we note easily that $\phi(z)$ is regular at least in the strip $|\operatorname{Im}(z)|<1$ and cannot have any zeros in this strip. For further investigation, we have to make a thorough study of the structure of the function $\cosh (\pi z / 2)$. We note that $\cosh (\pi z / 2)$ is an entire function of order unity having only simple zeros at the points.

$$
z= \pm i \alpha_{r}, \quad \text { where } \quad \alpha_{r}=2 r+1, \quad r=0,1,2, \cdots
$$

all situated on the imaginary axis. Thus we can write the canonical representation of $\cosh (\pi z / 2)$ as

$$
\cosh (\pi z / 2)=\prod_{r=-\infty}^{\infty}\left(1-\frac{z}{i \alpha_{r}}\right) e^{z / i \alpha_{r}}
$$

where

$$
\alpha_{ \pm r}= \pm \alpha_{r} \quad \text { and } \quad \alpha_{r}=2 r+1, \quad r=0,1,2, \cdots
$$

We also verify easily that the condition $\sum_{r=0}^{\infty} 1 / \alpha_{r}^{2}<\infty$ is satisfied. We now study a particular product representation of $\cosh (\pi z / 2)$. For this purpose, we partition the entire set of non-negative integers $(0,1,2, \cdots)$ into two mutually exclusive and exhaustive subsets and denote by

$$
(r)=\left(r_{1}, r_{2}, r_{3} \cdots\right)
$$

an arbitrary subset of non-negative integers, finite or denumerable, and by

$$
(s)=\left(s_{1}, s_{2}, s_{3}, \cdots\right)
$$

the corresponding complementary subset such that

$$
(r)+(s)=(0,1,2, \cdots) \text {. }
$$

As a special case, the set $(r)$ may constitute the whole set of non-negative integers $(0,1,2, \cdots)$. Then the set $(s)$ naturally reduces to an empty set. With the sets $(r)$ and $(s)$ so defined, we construct

$$
\text { and } \begin{aligned}
P(z) & =\prod_{j=1}^{\infty}\left(1-\frac{z}{i \alpha_{r_{j}}}\right) e^{z / i \alpha_{r_{i}}}, \\
Q(z) & =\prod_{j=1}^{\infty}\left(1-\frac{z}{i \alpha_{s_{j}}}\right) e^{z / i \alpha_{s_{j}}}
\end{aligned}
$$


It is then easy to verify that

$$
\cosh (\pi z / 2)=P(z) Q(z) P(-z) Q(-z) .
$$

We next study the following product representation of $\phi(z)$ as:

$$
\phi(z)=\frac{1}{P(z) Q(-z)} \theta(z)
$$

where $P(z)$ and $Q(z)$ are already defined in (3.3) and $\theta(z)$ is an analytic function of $z$ regular at least in the strip $|\operatorname{Im}(z)|<1$, and cannot have any zeros in that strip.

We discuss the analytical behavior of $\theta(z)$ for complex values of $z$. We have the equation

$$
\phi(z) \phi(-z)=\frac{1}{P(z) Q(-z)} \cdot \frac{1}{P(-z) Q(z)} \cdot \theta(z) \theta(-z) .
$$

Using (3.1) and (3.4), we get at once from (3.6)

$$
\theta(z) \theta(-z)=1 \text {. }
$$

We make use of the transformation $\psi(z)=\log \phi(z)$ and see easily that $\psi(z)$ is also regular at least in the same strip $|\operatorname{Im}(z)|<1$. It is now easy to verify from (3.7) that $\psi(z)$ is an odd function, that is $\psi(-z)=-\psi(z)$.

Thus $\psi(z)$ has the power series representation

$$
\psi(z)=\sum_{n=0}^{\infty} a_{2 n+1} z^{2 n+1}
$$

about the origin $z=0$ for all $z$ in the circle $|z|<1$. We next note that each of the functions $\phi(z)$ and $P(z) Q(-z)$ is real on the imaginary axis. Hence from (3.5) it follows at once that $\theta(z)$ and consequently $\psi(z)=\log \theta(z)$ must also be real for purely imaginary values of $z$. This easily gives that all the coefficients $a_{2 n+1}$ in (3.8) must be purely imaginary and thus we can write $\psi(z)$ $=i A(z)$ where $A(z)$ is an odd function and real on the real axis. With this notation, we can rewrite (3.5) in the form

$$
\phi(z)=\frac{1}{P(z) Q(-z)} \cdot e^{i \Lambda(z)} .
$$

Thus the problem of making a complete enumeration of the class of characteristic functions $\phi(z)$ which satisfy the relation (3.9) is essentially identical with that of determining all possible types of the function $A(z)$ such that $\phi(z)$ in (3.9) is a characteristic function. But this problem seems to be extremely difficult. In the present paper we shall conside $\cdot$ only some particular forms of the function $A(z)$ which makes $\phi(z)$ in (3.9) a characteristic function.

In the present section we shall consider in detail the particular case when 
$A(z)$ in (3.9) is an entire function, that is, when $\theta(z)$ in (3.5) is an entire function without any zeros. In such a case we prove first that the order of the entire function $\theta(z)$ cannot exceed unity. For this purpose we require a lemma on the decomposition of analytic characteristic functions which is due to Raikov [6].

LeMma 3.1. Let $f(z)$ be a decomposable analytic characteristic function and $f_{1}(z)$ a factor of $f(z)$. Let $f(-i v)$ exist for some $v \neq 0$, then for this $v, f_{1}(-i v)$ must also exist. Moreover, there exist two finite numbers $\alpha>0$ and $\beta \geqq 0$ not depending on $v$ for which the inequality

$$
f_{1}(-i v) \leqq \alpha \cdot e^{\beta|v|} f(-i v)
$$

is satisfied.

Then using the well-known property of the positive definite functions

$$
\max _{-\infty \leqq t \leqq \infty}\left|f_{1}(t+i v)\right| \leqq f_{1}(i v)
$$

we get at once from (3.10) the inequality

$$
\left|f_{1}(-z)\right| \leqq \alpha \cdot e^{\beta|v|} f(-i v)
$$

where $z=t+i v$ ( $t$ and $v$ both real). We now apply the inequality (3.11) to the relation (3.1) and get

$$
|\phi(z)| \leqq \alpha \cdot e^{\beta|v|} \cdot \frac{1}{\cos (\pi v / 2)}
$$

where $z=t+i v$ ( $t$ and $v$ both real). Then using the expression for $\phi(z)$ in (3.5) and substituting it in (3.12), we get

$$
\begin{aligned}
|\theta(z)| \cos (\pi v / 2) & \leqq \alpha \cdot e^{\beta|v|} \cdot|P(z) Q(-z)| \\
& \leqq \alpha \cdot e^{\beta|z|} \cdot|P(z) Q(-z)|
\end{aligned}
$$

where $z=t+i v$ ( $t$ and $v$ both real). But $P(z) Q(-z)$ is an entire function of order unity. Hence from (3.13) it follows at once that the order of the entire function $\theta(z)$ cannot exceed unity. Finally using the factorization theorem of Hadamard [8, p. 250] we have $\theta(z)=e^{i a z}(a$ real $)$. Thus we obtain

$$
\phi(-z)=\frac{1}{P(-z) Q(z)} e^{-i a z} .
$$

We next prove the following lemma.

Lemma 3.2. The function $\phi(-z)$ given in (3.14) is always a characteristic function.

Proof. We can write from (3.3) 


$$
\begin{aligned}
R(z) & =P(-z) Q(z) \\
& =\prod_{j=1}^{\infty}\left(1+\frac{z}{i \alpha_{r_{j}}}\right) \cdot e^{-z / i \alpha_{r_{j}}} \prod_{j=1}^{\infty}\left(1-\frac{z}{i \alpha_{\delta_{j}}}\right) e^{z / i \alpha_{s_{j}}} \\
& =\prod_{k=1}^{\infty}\left(1-\frac{z}{i \beta_{k}}\right) e^{z / i \beta_{k}}
\end{aligned}
$$

where the set $\left\{\beta_{k}\right\}$ is obtained by combining together the two sets $\left\{-\alpha_{r_{j}}\right\}$ and $\left\{\alpha_{\boldsymbol{s}_{j}}\right\}$. Clearly each $\beta_{k}$ is real and further $\sum_{\boldsymbol{k}=1}^{\infty} 1 / \beta_{\boldsymbol{k}}^{2}<\infty$. Thus $R(z)$ is an entire function of order unity and having only purely imaginary zeros. Hence applying the result $[4$, p. 140$]$ it follows at once that $1 / R(z)=1 / P(-z) Q(z)$ is always the characteristic function of a distribution. Next we note that in (3.14) the factor $e^{-i a z}$ ( $a$ real) is always the characteristic function of an improper distribution. This completes the proof of Lemma 3.2.

Finally we note that we can assume $a=0$ in (3.14) without any loss of generality, as this evidently means that the distribution function of $|x|$ is uniquely determined except for an arbitrary scale factor. Thus except for an arbitrary location parameter, we can write the characteristic function of the distribution of $-\log |z|$ as

$$
\phi(-z)=\frac{1}{P(-z) Q(z)}
$$

where $P(z)$ and $Q(z)$ are defined in (3.3).

We next discuss the arrangement of the elements in the sets $(r)$ and $(s)$, where it is given that the distribution function $F(x)$ has only finite absolute moments up to a certain order $k(k \geqq 1)$. Without any loss of generality we can assume that the integer elements in the sets $(r)$ and $(s)$ are arranged in the increasing order of magnitude such that $r_{1}<r_{2}<r_{3} \cdots$ and $s_{1}<s_{2}<s_{3} \cdots \cdots$ Then it follows easily from (3.15) that $\phi(-z)$ is an analytic characteristic function which is regular in the horizontal strip $-\alpha_{r_{1}}<\operatorname{Im}(z)<\alpha_{s_{1}}$ of the complex z-plane. Hence using a theorem of Lévy [3] on analytic characteristic functions, we get that for the analytic characteristic function $\phi(-z)$ the singularities nearest to the real axis in either half-plane must be located at the points $z=-i \alpha_{r_{1}}$ and $z=i \alpha_{s_{1}}$ on the imaginary axis. On the other hand, applying Lemma 2.3, we deduce that when the distribution function $F(x)$ has finite absolute moments up to order $k(k \geqq 1), \phi(-z)$ is an analytic characteristic function which is regular in the strip $-1<\operatorname{Im}(z)<k+1$, and using Lévy [3], its singularities nearest to the real axis in either half-plane must be the purely imaginary points on the boundary of its strip of regularity, that is, the points $z=-i$ and $z=i(k+1)$ on the imaginary axis. Finally remembering that $\alpha_{r}=2 r+1$, we deduce at once the conditions $r_{1}=0$ and $s_{1}=k / 2$ which must be an integer, that is, $k$ must be an even integer. Thus we write $k=2 m$ (where $m$ is an integer). Thus we have proved that under the 
condition that the distribution function $F(x)$ has finite moments up to a certain even order $2 m$, the arrangement of the elements in the sets $(r)$ and $(s)$ will be as follows, the first $m$ elements of the set $(r)$ must be $0,1,2, \cdots, m-1$ and the first element of the set $(s)$ must be $m$, that is,

$$
\begin{aligned}
(r) & =(0,1,2, \cdots, m-1, \cdots) \\
(s) & =(m, \cdots)
\end{aligned}
$$

while the arrangement of the remaining integers in the sets $(r)$ and $(s)$ are entirely arbitrary, the integer elements in each of the sets being arranged in ascending order of magnitude.

We now summarize the main result in the form of the following theorem:

THEOREM 3.1. Let the characteristic function of the distribution of $-\log |x|$ be given by

$$
\phi(-z)=\frac{1}{P(-z) Q(z)}
$$

where $P(z)$ and $Q(z)$ are defined as in (3.3), corresponding to the two mutually exclusive and exhaustive subsets $(r)$ and $(s)$ of non-negative integers. Let the elements in both the sets be arranged in increasing order of magnitude and further subject to the conditions given in (3.16). Any such construction of $\phi(-z)$ gives a distribution function $F(x)$ having finite moments up to order $2 m$ for which the quotient $x / y$ has the Cauchy law.

Thus we see that a class of infinitely many distribution functions $F(x)$ can be constructed each having finite moments up to order $2 m$ with the property that the quotient has the Cauchy distribution.

4. Further examples. In this section we shall construct another class of characteristic functions $\phi(z)$ which satisfy the relation (3.9) where $A(z)$ is not an entire function. For the construction of this class, we have to make use of a lemma which is essentially connected with the rational characteristic functions and proved by Lukacs [5].

LEMMA 4.1. Let

$$
\Phi(t)=\frac{(1+i t / w)(1+i t / \bar{w})}{(1-i t / a)(1-i t / v)(1-i t / \bar{v})}
$$

where

$$
v=a+i b ; \quad w=\alpha+i \beta \text { and } a>0, b>0, \alpha>0, \beta>0 .
$$

Then the function $\Phi(t)$ is always a characteristic function, if and only if, one of the following two conditions (which are mutually exclusive) holds:

(i) $\beta=\left\{b^{2}-(a+\alpha)^{2}\right\}^{1 / 2} \geqq 3^{1 / 2}(a+\alpha)$,

(ii) $\beta \neq\left\{\left\{b^{2}-(a+\alpha)^{2}\right\}^{1 / 2}\right.$ and simultaneously $\beta^{2} \geqq(a+\alpha)^{2}+b^{2} / 2$. 
The proof of this lemma is based on the properties of certain non-negative trigonometric polynomials and given in [5].

Here we are interested in only a special case of the above lemma when $w=v$. Thus we see easily that

$$
\Phi(t)=\frac{(1+i t / v)(1+i t / \bar{v})}{(1-i t / a)(1-i t / v)(1-i t / \bar{v})}
$$

where

$$
v=a+i b ; \quad \bar{v}=a-i b ; \quad a>0 ; \quad b>0
$$

is always the characteristic function of a distribution whenever $b \geqq 2^{3 / 2} a$.

We now select $\theta(z)$ in the equation (3.5) as follows:

$$
\theta(z)=e^{i A(z)}=\prod_{j=1}^{N_{1}} \frac{\left(1+\frac{z}{i \gamma_{r_{j}}}\right)\left(1+\frac{z}{i \bar{\gamma}_{r_{j}}}\right)}{\left(1-\frac{z}{i \gamma_{r_{j}}}\right)\left(1-\frac{z}{i \bar{\gamma}_{r_{j}}}\right)} \cdot \prod_{j=1}^{N_{2}} \frac{\left(1-\frac{z}{i \gamma_{s_{j}}}\right)\left(1-\frac{z}{i \bar{\gamma}_{s_{j}}}\right)}{\left(1+\frac{z}{i \gamma_{s_{j}}}\right)\left(1+\frac{z}{i \bar{\gamma}_{s_{j}}}\right)}
$$

where

$$
\begin{array}{ll}
\gamma_{r_{j}}=\alpha_{r}+i \beta_{r_{j}} ; & \beta_{r_{j}} \geqq 2^{3 / 2} \alpha_{r_{j}}, \\
\gamma_{s_{j}}=\alpha_{s_{j}}+i \beta_{s_{j}} ; & \beta_{s_{j}} \geqq 2^{3 / 2} \alpha_{s ;},
\end{array}
$$

$\alpha_{r_{j}}=2 r_{j}+1 ; \alpha_{s_{j}}=2 s_{j}+1$ corresponding to the elements of the sets $(r)$ and $(s)$ and the numbers $N_{1}$ and $N_{2}$ may be either finite or infinite. We further note that the condition

$$
\sum_{r=0}^{\infty} 1 /\left|\gamma_{r}\right|^{2}<\sum_{r=0}^{\infty} 1 / \alpha_{r}^{2}<\infty \text { is satisfied }
$$

We substitute this expression for $\theta(z)$ in (4.3) in the expression for $\phi(z)$ in (3.5) and get after a little rearrangement of terms

$$
\begin{aligned}
\phi(z) & =\prod_{=1}^{N_{1}} \frac{\left(1+\frac{z}{i \gamma_{r_{j}}}\right)\left(1+\frac{z}{i \bar{\gamma}_{r_{j}}}\right)}{\left(1-\frac{z}{i \alpha_{r_{j}}}\right)\left(1-\frac{z}{i \gamma_{r_{j}}}\right)\left(1-\frac{z}{i \bar{\gamma}_{r_{j}}}\right) e^{z / i \alpha_{r_{j}}}} \prod_{j=N_{1}+1}^{\infty} \frac{1}{\left(1-\frac{z}{i \alpha_{r_{j}}}\right) e^{i / i \alpha_{r_{j}}}} \\
& \times \prod_{j=1}^{N_{2}} \frac{\left(1-\frac{z}{i \gamma_{s_{j}}}\right)\left(1-\frac{z}{i \bar{\gamma}_{s_{j}}}\right)}{\left(1+\frac{z}{i \alpha_{s_{j}}}\right)\left(1+\frac{z}{i \gamma_{s_{j}}}\right)\left(1+\frac{z}{i \bar{\gamma}_{s_{j}}}\right) e^{-z / i \alpha_{s_{j}}}} \prod_{j=N_{2}+1}^{\infty} \frac{1}{\left(1+\frac{z}{i \alpha_{s_{j}}}\right) e^{-z / i \alpha_{\alpha_{j}}}} .
\end{aligned}
$$

We note that if both the numbers $N_{1}$ and $N_{2}$ are finite, it follows as an 
immediate application of (4.2) that $\phi(z)$ in (4.4) is a characteristic function, but if, on the other hand, either of the numbers $N_{1}$ and $N_{2}$ are infinite, it is also easy to verify that $\phi(z)$ is a characteristic function as a simple further consequence of the continuity theorem of Lévy. We can also see easily that when $\phi(z)$ is of the form (4.4), the characteristic function $\phi(-z)$ is an analytic characteristic function which is regular in the horizontal strip $-\alpha_{r_{1}}<\operatorname{Im}(z)$ $<\alpha_{s_{1}}$ (of course, under the assumption that the elements in both the sets $(r)$ and $(s)$ are arranged in increasing order of magnitude). Thus in this case also we can state a theorem which is analogous to the Theorem 3.1. Hence the characteristic function $\phi(z)$ constructed in (4.4) again generates a class of infinitely many distribution functions $F(x)$ with the desired property.

\section{REFERENCES}

1. R. G. Laha, An example of a non-normal distribution where the quotient follows the Cauchy law, Proc. Nat. Acad. Sci. (U.S.A.) vol. 44 (1958) pp. 222-223.

2. - On the laws of Cauchy and Gauss, Ann. Math. Statist. vol. 30 (1959), in press. 17-39.

3. P. Lévy, L'arithmétique des lois de probabilité, J. Math. Pures Appl. vol. 17 (1938) pp.

4. E. Lukacs and O. Szsáz, Certain Fourier transforms of distributions, Canad. J. Math. vol. 3 (1951) pp. 140-144.

5. E. Lukacs, Remarks concerning characteristic functions, Ann. Math. Statist. vol. 28 (1957) pp. 717-723.

6. D. A. Raikov, On the decomposition of Gauss and Poisson laws, Izv. Akad. Nauk. SSSR Ser. Mat. vol. 2 (1938) pp. 91-124 (in Russian).

7. G. P. Steck, $A$ uniqueness property not enjoyed by the normal distribution, Ann. Math. Statist. vol. 29 (1958) pp. 604-606.

8. E. C. Titchmarsh, The theory of functions, Oxford, 1939.

Catholic University of America, Washington, D. C.

Columbia University, NEw YORK, N. Y. 